\title{
Performance Evaluation of Soft Handover in WCDMA System
}

\section{S.A.Mawjoud}

\section{S.H.Fasola}

Electrical Eng. Dept. / University of Mosul

\begin{abstract}
In this paper soft handover parameters in WCDMA system are investigated. They include the effect of imperfect power control on the downlink capacity and power assignment to the mobile station, the micro diversity gain which represents the gain in the downlink direction, and the macro diversity gain which represents the gain in the uplink direction.

The results showed that the power control error increases the total power consumption by the mobile station which leads to decrease the capacity (number of users per cell). Soft handover decreases the fade margin which leads to improve the coverage area. During soft handover the mobile station needs lower signal to noise ratio compared with no soft handover to obtain the specific bit error rate.
\end{abstract}

Keyword: Cellular Mobile System, Soft Handover (SHO), Power Control.

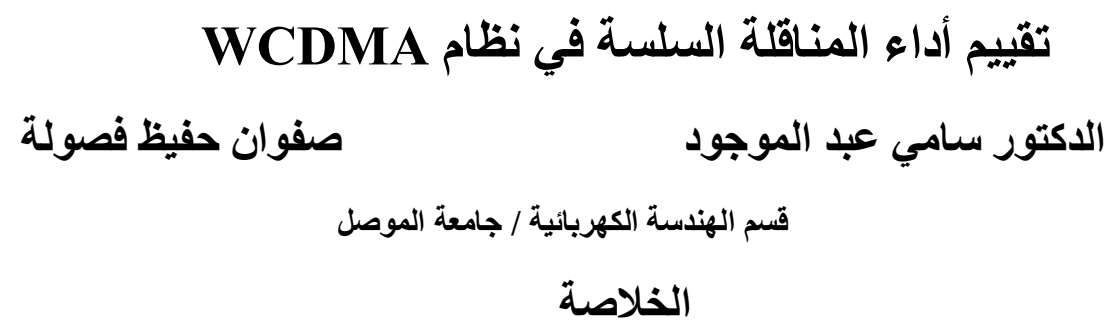

في هذا البحث تم تقييم معاملات المناقلة السلسة في نظام WCDMA. هذه المعاملات تثمل تأثلثير السيطرة

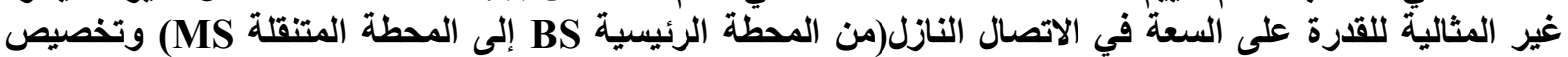

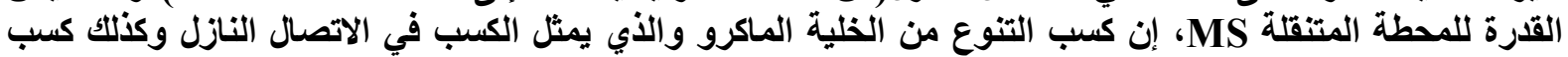

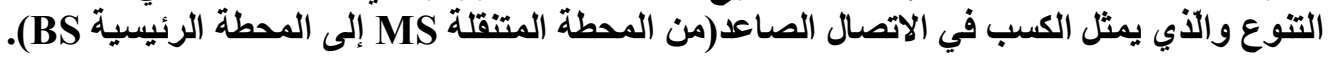

أظهرت النتائج إن الخطأ في السيطرة على القدرة يؤدي إلى الزيادة في استهلاك القدرة الكلية للمحطة

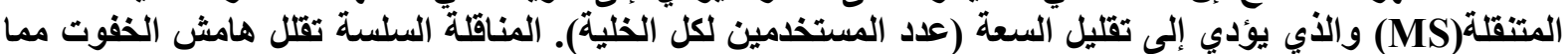

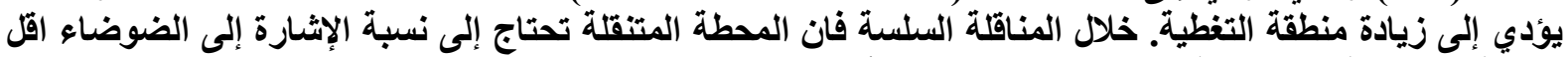

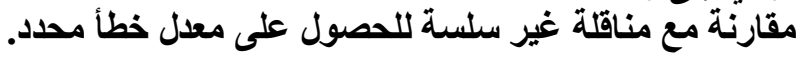




\section{Introduction:}

Mobility is the main advantage of mobile cellular systems. The ability to communicate anywhere, at any time was the main reason for great success of wireless communications in the 90's. Handover is the key concept in providing mobility. Handover, is the process of changing the channel (frequency, time slot, spreading code, or combination of them) associated with the current connection while a call is in progress. Handover is divided into two broad categories: hard and soft handovers. They are also characterized by "break before make" and "make before break", respectively. The first generation (1G) system employed Frequency Division Multiple Access (FDMA) technique. In the second generation(2G) employed Time Division Multiple Access (TDMA) technique, handover is performed by changing the carrier frequency used by a mobile station (MS) in the adjacent cell, this type of handover is called hard handover (HHO). In the third generation (3G), Code Division Multiple Access (CDMA) systems use soft handover (SHO) procedure because all cells share the same uplink (UL) and downlink (DL) frequency[1][2][3].

\section{Basic Principle of Soft Handover:}

Soft handover is a technique that allows wireless user equipment to stay connected to several base stations(BSs) in Wideband Code Division Multiple Access(WCDMA) system. CDMA technique makes possible the ability to maintain a previous connection while adding a new one (make before break)[4]. The soft handover process is different in different transmission directions. In the uplink direction (UL) the MS transmits signals to air through its Omni directional antenna. Two BSs (or three BSs) in the active set(active set is the list of cells that currently have connections with MS) receive the signals at the same time due to the frequency reuse factor of one in CDMA system, then the signals are sent forward to the radio network control (RNC) for selection combining. The strongest signal is considered and the other is discarded. As a result, in the UL direction, there is no extra channel required to support soft handover process. But in the downlink (DL), this is not the case, when two or three BSs are transmitting simultaneously, the MS simply combine the signals from different BSs since it sees them as just additional multipath components[5]. For this purpose, the MS containing the RAKE receiver and its fingers process the received signals[3]. During the soft handover, the MS communicates with two or more adjacent cells each has a BS and these BSs are controlled by the same RNC (intraRNC) or different RNCs (inter-RNC). To support soft handover in the downlink direction, at least one extra downlink channel (2-way soft handover) is required. Therefore, to support soft handover in the downlink, more resources are essential[5].

\section{Propagation Path Loss:}

[6]:

Typically in radio channel models, three effects contribute to the loss of a radio link

1) Path loss attenuation with respect to distance.

2) Shadow fading (slow fading).

3) Multipath fading (fast fading).

Path loss is the decrease of the received power with distance due to reflection, diffraction around structures, and refraction. Shadowing occurs due to obstruction in the line of site (LOS) path between transmitter and receiver by buildings, hills, trees, and foliage. 
Multipath fading occurs in a situation in which the transmitted signal reaches the receiver over multiple paths. The sum of multipath signal results in fading dips[6][7][8].

The propagation loss is generally modeled by the following equation[9].

$\mathrm{L}(\mathrm{r}, \zeta)=\mathrm{r}^{\mathrm{m}} \cdot 10^{\zeta / 10}$

where " $r$ " is the distance between the BS and the MS, " $m "$ is the path loss exponent which indicates the rate at which the path loss increases with distance. The value of " $m$ " depends on the specific propagation environment $(2<m<4)$ typical value of 4 in urban area [10]. " $\zeta$ " is the attenuation in decibel due to shadowing which is a log-normal random variable with zero mean and standard deviation " $\sigma$ " which is independent of distance, it ranges from 5 to 12 with typical value of $8 \mathrm{~dB}$. The fast fading loss is not included in this model because it can be processed by the RAKE receiver [3][7].

The random component of shadowing may be expressed as the sum of two components: the first is due to the near field of the user that is common to all BSs, and the second pertains to the receiving base station and is independent for each BSs [9].

$\zeta i=a \xi+b \xi i$

$a^{2}+b^{2}=1$

where $i$ is the number of interfering BS's.

where " $a$ " and " $b$ " are constants.

$E(\zeta i)=E(\xi)=E(\xi i)=0$ for all $i$, where $\mathrm{E}()$ is the expected value.

$\operatorname{Var}(\zeta \mathbf{i})=\operatorname{Var}(\xi)=\operatorname{Var}(\xi \mathbf{i})=\sigma^{2}$ for all $i$

$E(\xi \xi i)=0$ for all $i$

$E(\xi i \xi j)=0$ for all $i \neq j$.

The normalized correlation coefficient of the loss due to two BSs $i$ and $j$ is given by[9]:

$\left(\frac{E(\xi i \xi j)}{\sigma^{2}}\right)=a^{2}=1-b^{2}$

$a^{2}=b^{2}=1 / 2$ for normalized correlation between BSs.

\section{System Scenario:}

The system scenario consists of seven-cell hexagonal grid, as shown in figure (1). It consists of serving $\mathrm{BS}$ at the center and surrounded by six interfering BSs (first tire). Near the cell boundaries, the shaded area represents the soft handover region (also called soft handover zone) and the remaining is known as the non-soft handover region. All the mobiles in shaded areas are in soft handover status, communicating with two or more base stations simultaneously.

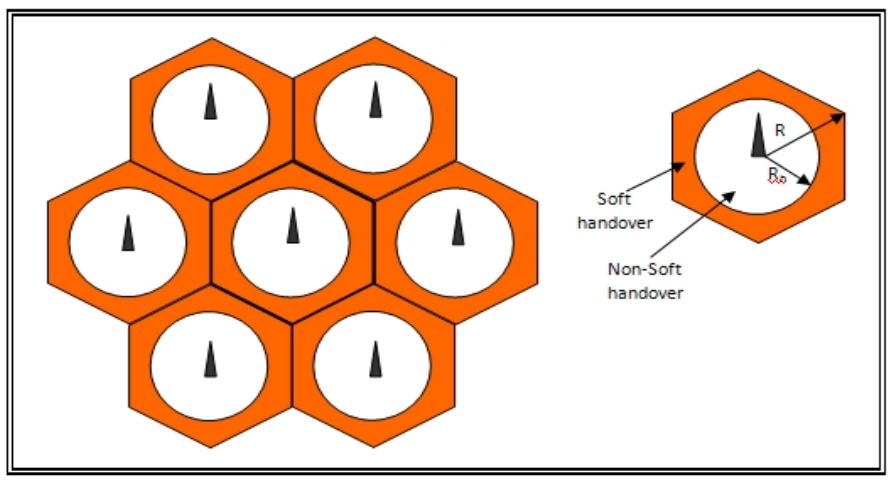

Figure 1 System Scenario

Figure (1). System scenario.

Assuming that interference coming from the second tire can be neglected. The ratio between soft handover region and the entire cell area is called soft handover overhead [11]. 


\section{Downlink Soft Handover Power Allocation and Capacity Estimation Under Different Power Control:}

- Perfect Power Control:

In WCDMA system, power control is one of the crucial functionalities for radio resource management. Unlike that in the

uplink, the motivation for having power control in the downlink is not due to the nearfar problem but is due to one BS to many MSs. Perfect power control in the downlink equalizes the received bit energy to interference power spectral density $\left(E_{b} / I_{o}\right)$ of all MSs at a target value at all times and distance [7]. The $E_{b} / I_{o}$ can be expressed without soft handover as [11]:

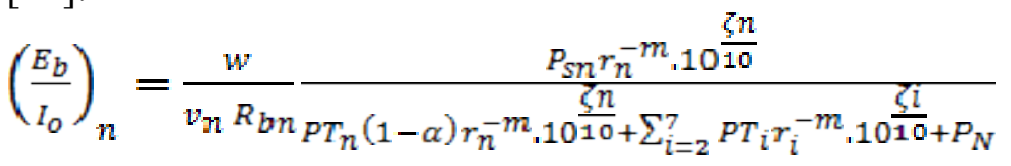

where $\left(E_{b} / I_{o}\right)_{n}$ is the bit energy to interference power spectral density for user $n, w$ is the chip rate, $R_{b n}$ is the bit rate for user $n$, which depends on the type of service, $v_{n}$ is the activity factor for user $n, P_{s n}$ is the required transmit power at $B S_{n}$ allocated for user $n, P T_{n}$ is the maximum transmit power of the serving base station $\left(B S_{n}\right), P T_{i} \quad$ is the total transmit power of interfering BS's $\left(B S_{i}\right)$ where $2 \leq i \leq 7, r_{n}^{-m} .10^{\frac{\xi n}{10}}$ is the propagation loss between serving $\mathrm{BS}\left(\mathrm{BS}_{\mathrm{n}}\right)$ and user $n, r_{i}^{-m} \cdot 10^{\frac{\zeta i}{10}}$ is the propagation loss between interfering $\mathrm{BS}_{\mathrm{i}}$ and user $n, \alpha$ is the orthogonality factor and $P_{N}$ is the background noise power at the receiver.

The background noise power is negligible compared to the total interference received from all base stations $\left(P T_{i} r_{i}^{-m} \cdot 10^{\frac{\zeta i}{10}}>P_{N}\right)[11]$. Assuming that all users have the same bit rate and all $\mathrm{BSs}$ transmit the same power level $\left(P T_{n}=P T_{i}\right)$, then:

$\left(\frac{E_{b}}{I_{Q}}\right)_{n}=\frac{w}{v R_{b}} \frac{P_{S T}}{\left[(1-\alpha)+\sum_{i=2}^{7}\left(\frac{r_{i}}{r_{n}}\right)^{-m} \cdot 10^{\frac{\zeta \bar{c}-\zeta n}{10}}\right]}$

When an MS is in the soft handover status, all the BSs in the active set(AS) need to allocate proper power for the downlink channels communicating with the MS. In the case of 2-way SHO (BS1 and $\mathrm{BS}_{2}$ ), obtaining maximum ratio combining (MRC) in the receiver, therefore the received $\mathrm{E}_{\mathrm{b}} / \mathrm{I}_{\mathrm{o}}$ is given by[2]:

$\left(\frac{E_{b}}{I_{o}}\right)=\left(\frac{E_{b}}{I_{o}}\right)_{1}+\left(\frac{E_{b}}{I_{o}}\right)_{2}$

From equation $3,\left(E_{b} / I_{o}\right)_{1}$ and $\left(E_{b} / I_{o}\right)_{2}$ can be found and hence $\left(\frac{E_{b}}{I_{o}}\right)$ is:

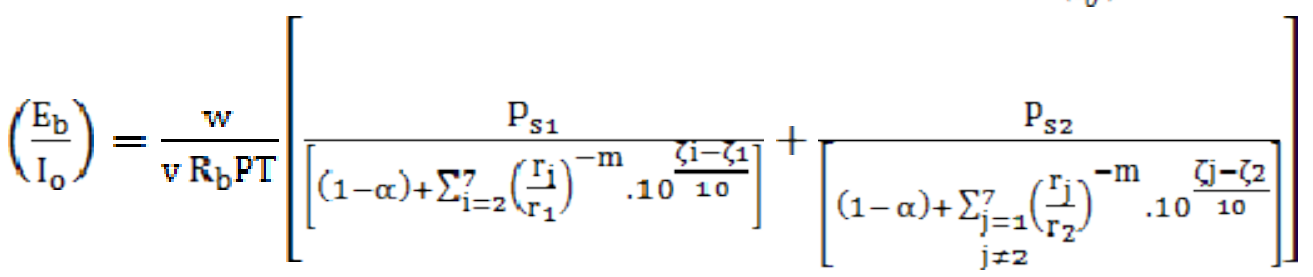

Assuming balance power control $\mathrm{P}_{\mathrm{s} 1}=\mathrm{P}_{\mathrm{s} 2}$ [2]. The total transmit power dedicated to MS under 2-way SHO can be written as: 


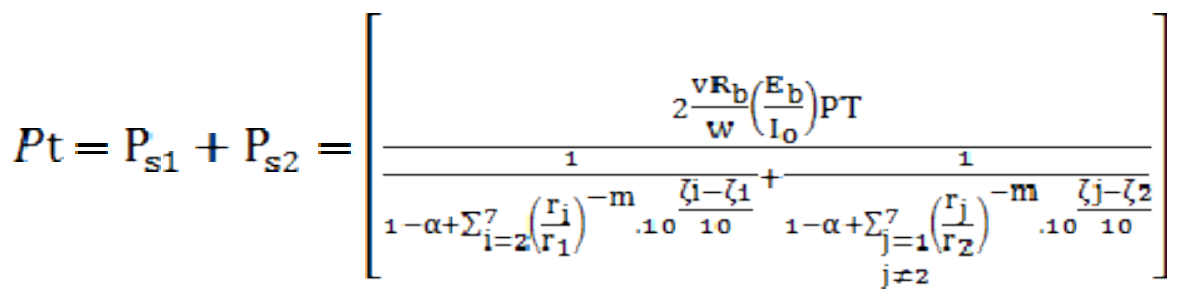

where $P t$ represents the total required power for the mobile under 2-way SHO.

The total transmit power from BS is composed of power for common control channel and the sum of power for each downlink dedicated channel [7].

$P T=P T \mu+\sum_{n=1}^{N} P_{s n}$

where $\mu$ is the ratio of common control channel power to the total transmit power of the BS and $N$ is the number of active users within each cell.

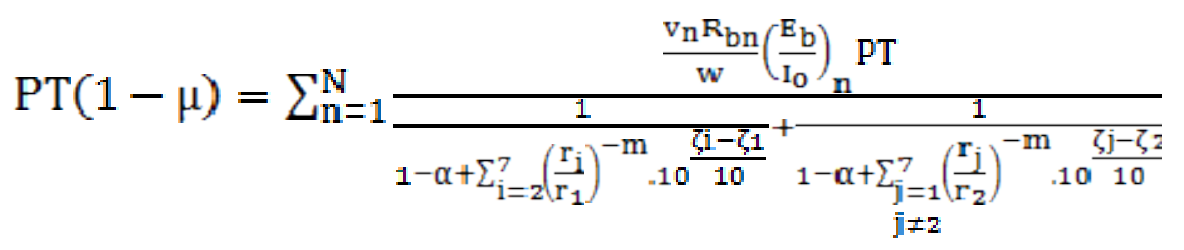

In this case the capacity under perfect power control when the MS is connected with two BSs can be expressed as:[12]

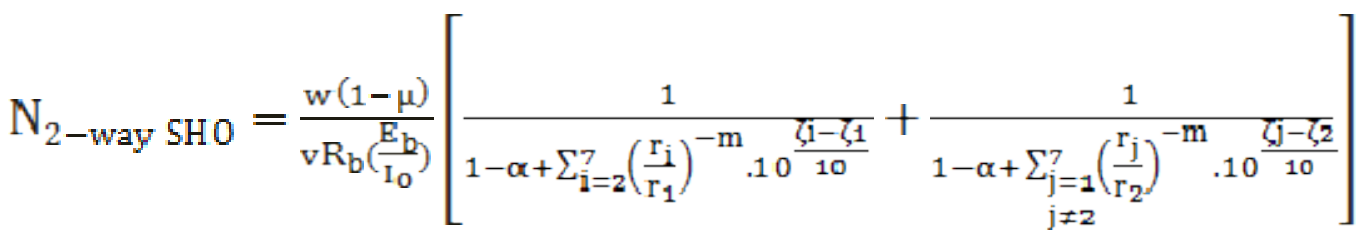

The same procedure can be followed to calculate $\left(\frac{E_{b}}{I_{o}}\right)$ for the 3-way SHO (the active set consists of three base stations BS1, BS2 and BS3)

$$
\left(\frac{E_{b}}{I_{0}}\right)=\left(\frac{E_{b}}{I_{0}}\right)_{1}+\left(\frac{E_{b}}{I_{0}}\right)_{2}+\left(\frac{E_{b}}{I_{0}}\right)_{3}
$$

The total transmit power dedicated to the MS under 3-way SHO can be written as[3]:

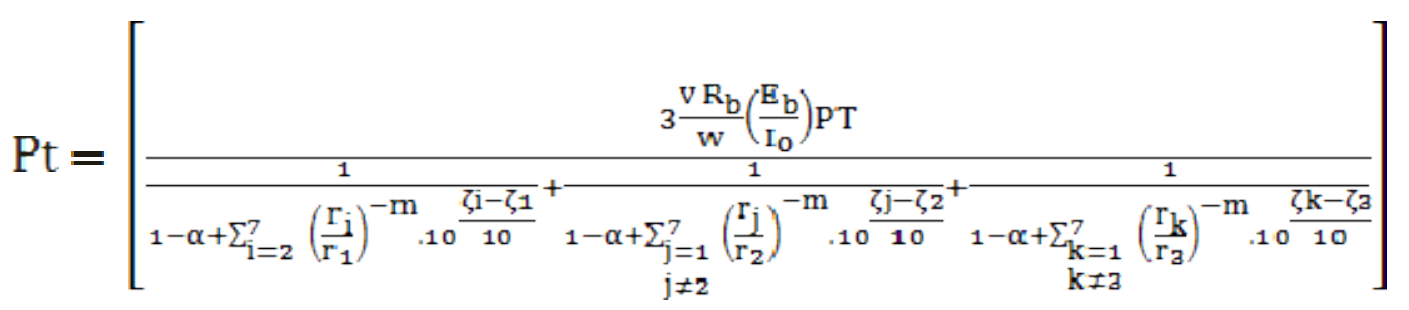

The number of active users within each cell under 3-way SHO is[12]: 


$$
\begin{aligned}
& \mathrm{N}_{3-\text { way } \mathrm{SHO}}=\frac{\mathrm{w}(1-\mu)}{\mathrm{v} \mathrm{R}_{\mathrm{b}}\left(\frac{\mathrm{E}_{\mathrm{b}}}{\mathrm{I}_{\mathrm{o}}}\right)}\left[\frac{1}{1-\alpha+\sum_{\mathrm{i}=2}^{7}\left(\frac{\mathrm{r}_{\mathrm{j}}}{\mathrm{r}_{1}}\right)^{-\mathrm{m}} \cdot 10^{\frac{\zeta_{\mathrm{i}}-\zeta 1}{10}}}+\frac{1}{1-\alpha+\sum_{\substack{\mathrm{j}=1 \\
\mathrm{j} \neq 2}}^{7}\left(\frac{\mathrm{r}_{\mathrm{j}}}{\mathrm{r}_{2}}\right)^{-\mathrm{m}} \cdot 10^{\frac{\zeta_{j}-\zeta_{2}}{10}}}+\right. \\
& \left.\frac{1}{1-\alpha+\sum_{\substack{\mathrm{k}=1 \\
\mathrm{k} \neq 3}}^{7}\left(\frac{\mathrm{r}_{\mathrm{k}}}{\mathrm{r}_{\mathrm{3}}}\right)^{-\mathrm{m}} \cdot 10^{\frac{\zeta \mathrm{k}-\zeta_{\mathrm{a}}}{10}}}\right]
\end{aligned}
$$

\section{Imperfect Power Control:}

In a practical system, the power control is not perfect. So, the power received by the MS from its serving BS will differ from the target power level $P_{s}$ by $\zeta_{e} \mathrm{~dB}$. This power control error $(\mathrm{PCE}) \zeta_{e}$ is a random variable with zero mean and standard deviation $\sigma_{e}$. The standard deviation $\sigma e$ reflects the degree of imperfection. With an assumption of zero standard deviation $\sigma e$, one will have perfect power control. There are several reasons for $\zeta_{e}$ being nonzero, such as the power measurement error at the MS and the inability to adjust the BS transmitted power sufficiently fast to force $\zeta_{e}$ to zero. $\zeta_{e}$ range is 1 to $4 \mathrm{~dB}$ [7].

The transmitted power for imperfect power control error from the BS at its MS can be expressed as in[7]:

$$
\mathrm{P}_{\text {simp }}=\mathrm{P}_{\mathrm{s}^{*}} 10^{\zeta_{\theta} / 10}, P_{\text {simp }}=P_{S}+\zeta_{e} \quad[\mathrm{~dB}]
$$

Substituting (13) into (6) and (11), the actual transmit power under imperfect power control situation with 2-way SHO and 3-way SHO respectively can be obtained as[12]:

$$
\begin{aligned}
& P_{\operatorname{simp}}=\frac{10^{\frac{\zeta \mathrm{e}}{10}} \frac{\mathrm{vR}_{\mathrm{b}}}{\mathrm{w}}\left(\frac{\mathrm{E}_{\mathrm{b}}}{\mathbf{I}_{0}}\right) \mathrm{PT}}{1-\alpha+\sum_{\mathrm{i}=2}^{7}\left(\frac{\mathrm{r}_{\mathrm{j}}}{\mathrm{r}_{1}}\right)^{-\mathrm{m}} \cdot 10^{\frac{\zeta_{1}-\zeta_{1}}{10}}+\frac{1}{1-\alpha+\sum_{\substack{j=1 \\
j \neq 2}}^{7}\left(\frac{\mathrm{r}_{\mathrm{j}}}{\mathrm{r}_{2}}\right)^{-\mathrm{m}} \cdot 10^{\frac{\zeta_{j}-\zeta_{2}}{10}}}}
\end{aligned}
$$

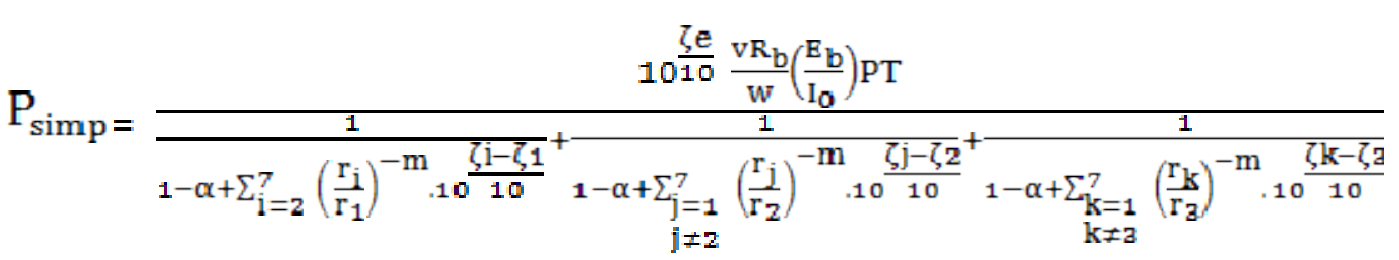

From equation (9) and (12) the capacity under imperfect power control when the MS connected with two BSs and three BSs respectively can be expressed as:

$$
N_{2-\text { way SHO }} \frac{w(1-\mu)}{10^{\zeta \ell / 10} v R_{b}\left(\frac{E_{b}}{I_{Q}}\right)}\left[\frac{1}{1-\alpha+\sum_{i=2}^{7}\left(\frac{r_{i}}{r_{1}}\right)^{-m} \cdot 10^{\frac{\zeta i-\zeta 1}{10}}} \frac{1}{1-\alpha+\sum_{\substack{j=1 \\ j \neq 2}}^{7}\left(\frac{r_{j}}{r_{2}}\right)^{-m} \cdot 10^{\frac{\zeta j-\zeta 2}{10}}}\right]
$$




$$
\begin{aligned}
& \mathrm{N}_{3-w a y} \mathrm{SHO}=\frac{\mathrm{w}(1-\mu)}{10 \frac{\zeta^{\mathrm{e}}}{10} \mathrm{v} \mathrm{R}_{\mathrm{b}}\left(\frac{\mathrm{E}_{\mathrm{b}}}{\mathrm{I}_{0}}\right)}\left[\frac{1}{1-\alpha+\sum_{\mathrm{i}=2}^{7}\left(\frac{\mathrm{r}_{\mathrm{i}}}{\mathrm{r}_{1}}\right)^{-\mathrm{m}} \cdot 10^{\frac{\zeta_{1}-\zeta_{1}}{10}}}+\frac{1}{1-\alpha+\sum_{\substack{\mathrm{j}=1 \\
\mathrm{j} \neq 2}}^{7}\left(\frac{\mathrm{r}_{\mathrm{j}}}{\mathrm{r}_{2}}\right)^{-\mathrm{m}} .10 \frac{\zeta_{\mathrm{j}}-\zeta_{2}}{10}}+\right. \\
& \left.\frac{1}{1-\alpha+\sum_{\substack{k=1 \\
k \neq 2}}^{7}\left(\frac{r_{k}}{r_{3}}\right)^{-m} \cdot 10^{\frac{\zeta k-\zeta, 3}{10}}}\right]
\end{aligned}
$$

\section{Macro Diversity Gain:}

Soft handoff provides gain against slow fading or shadowing which is either natural or man-made large structures which provoke the signal strength. The macro diversity gain appears because the received signal by the MS from BS1 and BS2 are not correlated [13]. Fade margin arises due to effects of shadow fading so the signal in the coverage area will drop below specific values. In order to overcome this, MS should raise transmission power by a certain amount. The amount by which transmission power should be raised is called fade margin [2]. Without shadow fading, the minimum power required is simply $10 \mathrm{~m} \log (\mathrm{r})$, where " $r$ " is the distance from the mobile to the serving base station and " $m$ " is the path loss exponent. However, with shadow fading, it is impossible to guarantee that the received power is always greater than the BS threshold value because of the tail of the Gaussian distribution which extends to infinity. Therefore, the quality requirement (bit error rate) is usually lowered such that the received power is guaranteed to be greater than the threshold, except for connection failure (outage probability). To ensure this, a fade margin is added to the transmit power. The outage probability in the system is defined as the probability of a call dropping before it is terminated and is denoted by $P_{\text {out }}[14]$.

The macro diversity gain is often defined as in [15]. If $\varphi_{1}$ is the shadow fade margin $(\mathrm{dB})$ required for one $\mathrm{BS}$ (no $\mathrm{SHO}), \varphi_{2}$ is the shadow fade margin (dB) required for two potential neighboring base stations (SHO allowed). Then the macro diversity gain is: $\varphi_{1-} \varphi_{2}$ $(\mathrm{dB})$.

From [9] the outage probability when the MS is connected with one base station $\left(\mathrm{BS}_{1}\right)$ is defined as:

$P_{\text {out }}=\operatorname{Pr}\left(10 m \log \left(r_{1}\right)+\zeta>\varphi\right)$

where $\varphi$ is the shadow fade margin[dB], and $\zeta$ is the attenuation in decibel due to shadowing.

$P_{\text {out }}=\frac{1}{\sqrt{2 \pi}} \int_{\zeta}^{\infty} e^{-\left(\frac{\zeta}{\sqrt{2} \sigma}\right)^{2}} d \zeta=Q\left(\frac{\varphi-10 m \log \left(r_{1}\right)}{\sigma}\right)$

With SHO an MS can be connected to more than one BS simultaneously. The RNC can choose the signal that has better reception quality. This is called selection diversity. An outage occurs when both signals are of unacceptable quality. Thus, the outage probability when the MS is connected with two base stations (BS1 and BS2) is [9][16]:

$P_{\text {out }}=P_{r}\left\{\operatorname{Min}\left[10 m \log \left(r_{1}\right)+\zeta_{1}, 10 m \log \left(r_{2}\right)+\zeta_{2}>\varphi\right]\right\}$ 
Since $\zeta_{1}$ and $\zeta_{2}$ are correlated according to their definition in equation (1), they can be expressed in terms of independent variables $\xi$, $\xi_{1}$ and $\xi_{2}$ as:[9]

$P_{\text {out }}=P_{r}\left\{\operatorname{Min}\left[10 \operatorname{mlog}\left(r_{1}\right)+b \xi_{1}+a \xi, 10 m \log \left(r_{2}\right)+b \xi_{2}+a \xi>\varphi\right]\right\}=$

$\frac{1}{\sqrt{(2 \pi \sigma)^{2}}} \int_{-\infty}^{\infty} \mathrm{e}^{-\left(\frac{\xi^{2}}{2 \sigma^{2}}\right)} \mathrm{d} \xi \int_{\xi_{1}}^{\infty} \mathrm{e}^{-\left(\frac{\xi_{1}^{2}}{2 \sigma^{2}}\right)} \mathrm{d} \xi_{1} \int_{\xi_{2}}^{\infty} \mathrm{e}^{-\left(\frac{\xi_{2}{ }^{2}}{2 \sigma^{2}}\right)} \mathrm{d} \xi_{2}=$

$\frac{1}{\sqrt{2 \pi}} \int_{-\infty}^{\infty} e^{-\left(\frac{\xi^{2}}{2 \sigma^{2}}\right)} Q\left(\frac{\varphi-10 m \log \left(r_{1}\right)-a \sigma \xi}{b \sigma}\right) Q\left(\frac{\varphi-10 m \log \left(r_{2}\right)-a \sigma \xi}{b \sigma}\right) d \xi$

The same procedure is used when the MS is connected with three base stations $\left(\mathrm{BS}_{1}\right.$, $\mathrm{BS}_{2}$, and $\left.\mathrm{BS}_{3}\right)$.

$P_{\text {out }}=\frac{1}{\sqrt{2 \pi}} \int_{-\infty}^{\infty} e^{-\left(\frac{\xi^{2}}{2 \sigma^{2}}\right)} Q\left(\frac{\varphi-10 m \log \left(r_{1}\right)-a \sigma \xi}{b \sigma}\right) Q\left(\frac{\varphi-10 m \log \left(r_{2}\right)-a \sigma \xi}{b \sigma}\right) *$
$Q\left(\frac{\varphi-10 m \log \left(r_{3}\right)-a \sigma \xi}{b \sigma}\right) d \xi$

\section{Micro Diversity Gain:}

Soft Handover gives an additional macro diversity gain against fast fading by reducing the required $E_{b} / I_{o}$ relative to a single radio link, because the fast fading from the two transmit BSs is uncorrelated. This diversity gain is usually referred to as micro diversity gain in order to avoid confusions with the macro diversity gain. The mobile can coherently combine the signals from the different BSs that are included in the active set since it sees them as additional multipath components. Normally Maximum Ratio Combining (MRC) technique is used, which provides additional macro diversity benefit [13].

The micro diversity gain is defined as the reduction of the required $E_{b} / I_{o}$ in SHO to obtain the required bit error rate $(B E R)$ when compared to the required $E_{b} / I_{o}$ in a single radio link [17]. Assuming independent $B E R$ for the different connections of a single MS, the overall $B E R$ of MS can be derived as [18]:

$B E R=\prod_{k \in A S}\left(B E R_{k}\right)$

For an MS in two way SHO, if the BER on the first connection is $1 \%$ and on the second connection is $20 \%$, then the total BER seems at the MS is $1 \% * 20 \%=0.2 \%$. The required $E_{b} / I_{o}$ can be reduced such that the total effective $B E R$ increases to $1 \%$ [15].

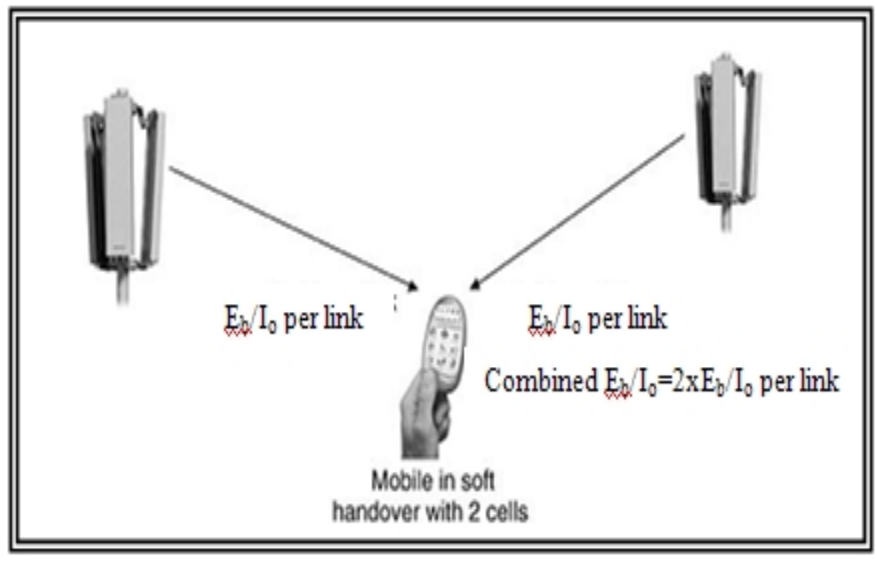

Figure (2). Soft handover modeling with two cells [11] 
For no SHO (MS connected with one BS) the average BER over fading channel for coherent PSK modulation can be expressed as [10]:

$B E R_{\text {no sho }}^{\text {fad. }}=\frac{1}{2}\left[1-\sqrt{\frac{\Gamma}{1+\Gamma}}\right]$

where $\Gamma=\left(E_{b} / I_{o}\right) \overline{\alpha^{2}}$ is the average value of the signal to noise ratio $(S N R)$ and $\overline{\alpha^{2}}$ is the mean fading power. For $\overline{\alpha^{2}}=1$, note that $\Gamma$ corresponds to the average $E_{b} / I_{o}$ for the fading channel.

For the MS in SHO status, the signals from the base stations are combined together using the $M R C$. Thus the average BER when the MS in SHO status can be expressed as [10]:

$B E R_{\text {she }}^{\text {fad }}=\left[\frac{1}{2}\left(1-\sqrt{\frac{I}{1+\Gamma}}\right)\right]^{M} \sum_{k=0}^{M-1}\left(\begin{array}{c}M-1+k \\ k\end{array}\right)\left[\frac{1}{2}\left(1 \sqrt{\frac{I}{1+\Gamma}}\right)\right]^{k}$

Where $M$ is the number of the BSs in the active set(AS). [13]:

The power level difference $(\Delta P)$ between the two base stations can be expressed as

$\Delta P[d B]=10 \log \left(\frac{\Gamma_{1}}{r_{2}}\right)$

\section{Results and Discussions:}

In this section, simulation programs are using MATLAB V. 7.4 are developed and used. Table (1). List the parameters used in the simulation [11] [3] [7].

Table 1 Parameters Used in the Simulation

\begin{tabular}{|c|c|}
\hline Parameters & Value \\
\hline Total power of the base station $(P T)$ & $20 \mathrm{~W}$ \\
\hline Chip rate $(W)$ & $3.84 \mathrm{Mcps}$ \\
\hline Orthogonality factor $(\alpha)$ & 0.6 \\
\hline Path loss exponent $(m)$ & 4 \\
\hline Standard deviation of shadowing $(\sigma)$ & $8 \mathrm{~dB}$ \\
\hline $\begin{array}{c}\text { The ratio of common control channel to the } \\
\text { total transmit power }(\mu)\end{array}$ & 0.2 \\
\hline Cell radius $(R)$ & $1 \mathrm{~km}$ \\
\hline Bit rate $\left(\mathrm{R}_{\mathrm{b}}\right)$ & $12.2 \mathrm{kbps}$ \\
\hline $\mathrm{E}_{\mathrm{b}} / \mathrm{I}_{\mathrm{o}}$ & $5 \mathrm{~dB}$ \\
\hline
\end{tabular}

Figure $(3 \mathrm{a}, \mathrm{b})$ illustrates the transmitted power from two BSs (2-way SHO), and three $\mathrm{BSs}(3$-way $\mathrm{SHO}$ ) to the MS respectively versus normalize distance for different PCE. For perfect power control $(\mathrm{PCE}=0 \mathrm{~dB})$ the MS consumes minimum power in order to overcome the loss due to shadowing, distance and a specific bit rate. The MS consumes more power compared to perfect power control error by $5 \%$ to $133 \%$ when PCE is increased to $1 \mathrm{~dB}$ and 
$4 \mathrm{~dB}$ respectively. For 3-way $\mathrm{SHO}$ the power needed is larger than 2-way SHO for the same PCE.

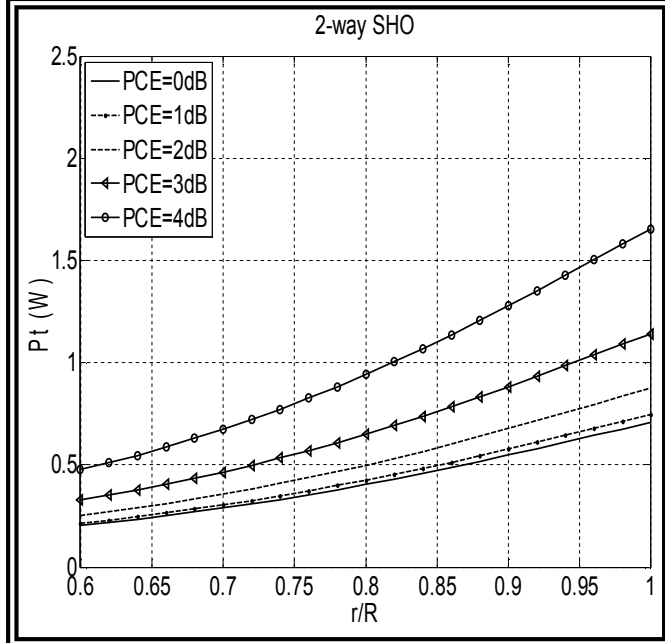

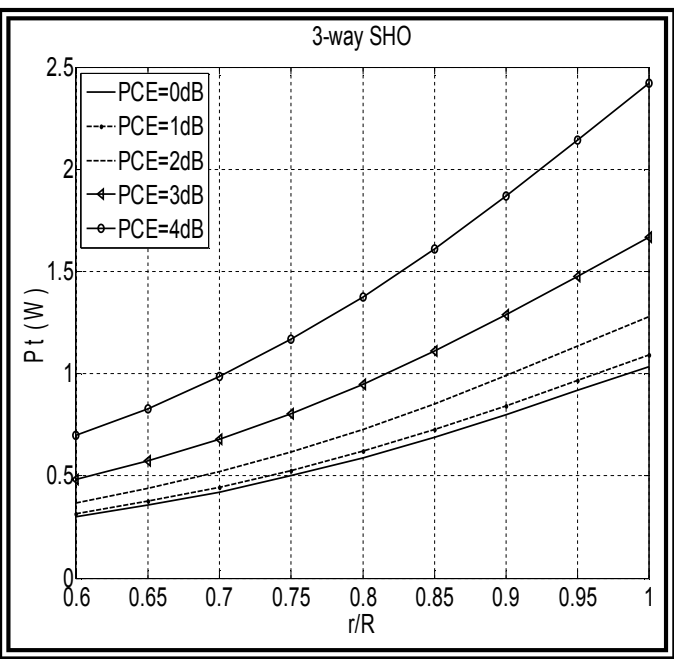

b

Figure (3). Total power required for the MS versus normalized cell radius

Figure $(4 a, b)$ illustrates the number of users per cell which can be supported versus distance for 2-way SHO and 3-way SHO respectively under different power control errors. Increasing PCE will increase the power consumption by MS which leads to increase the interference and decrease capacity (number of users per cells). The reduction in capacity due to imperfect power control is $5.2 \%$ to $57 \%$ when $\mathrm{PCE}=1 \mathrm{~dB}$ and $4 \mathrm{~dB}$ respectively. The number of users per cell increases for 3-way SHO compared to 2-way SHO for the same $\mathrm{PCE}$, this is because the power transmitted from one BS for 3-way SHO is lower compared with the power transmitted from one BS for 2-way SHO.

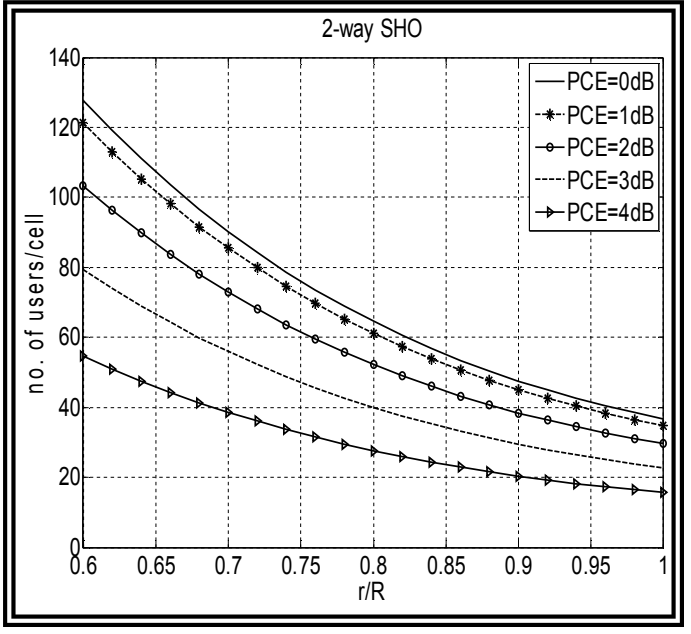

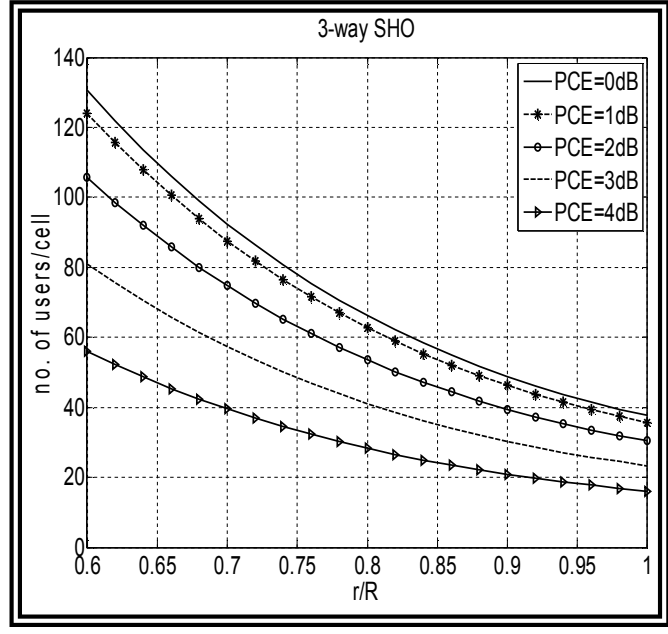

$\mathrm{b}$

Figure 4 Number of users per cell for versus normalized cell radius

Figure 5a illustrates the relation between fade margin and outage probability when the user is at the cell corner ( $\mathrm{r}=\mathrm{R})$, which represents the worst case, for no SHO and 2-way SHO. For a fixed value of outage probability, for instance 0.1 the fade margin required to obtain this value without $\mathrm{SHO}$ is $10.25 \mathrm{~dB}$, while in the case of 2-way SHO the fade margin required is $3.8 \mathrm{~dB}$, when $\mathrm{a}^{2}=0$ (where $\mathrm{a}^{2}$ is cross correlation coefficient between two BSs), and when $\mathrm{a}^{2}=0.5$ the fade margin required is $6 \mathrm{~dB}$. Figure $5 \mathrm{~b}$ illustrates the relation between fade 
margin and Pout, when the MS is with 3-way SHO compared without SHO. For a fixed value of Pout, for instance 0.1 , with 3-way SHO the required fade margin is $9.55 \mathrm{~dB}$ and $5.95 \mathrm{~dB}$ lower for $\mathrm{a}^{2}=0$ and $\mathrm{a}^{2}=0.5$ respectively.

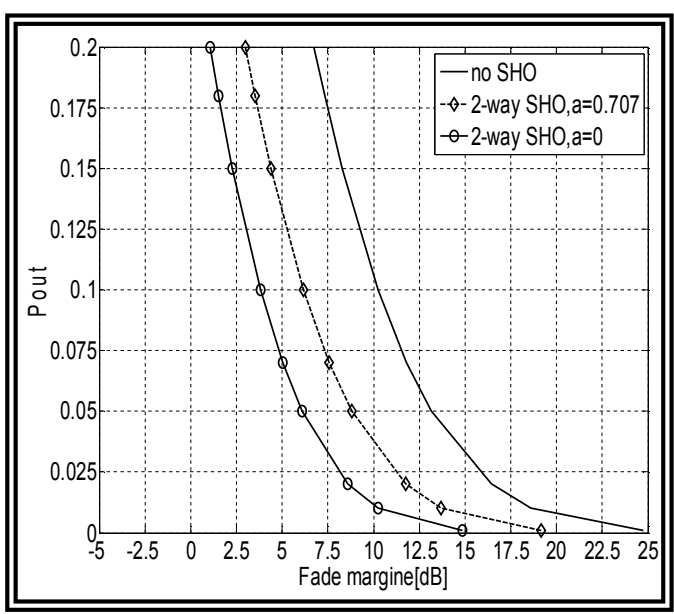

a

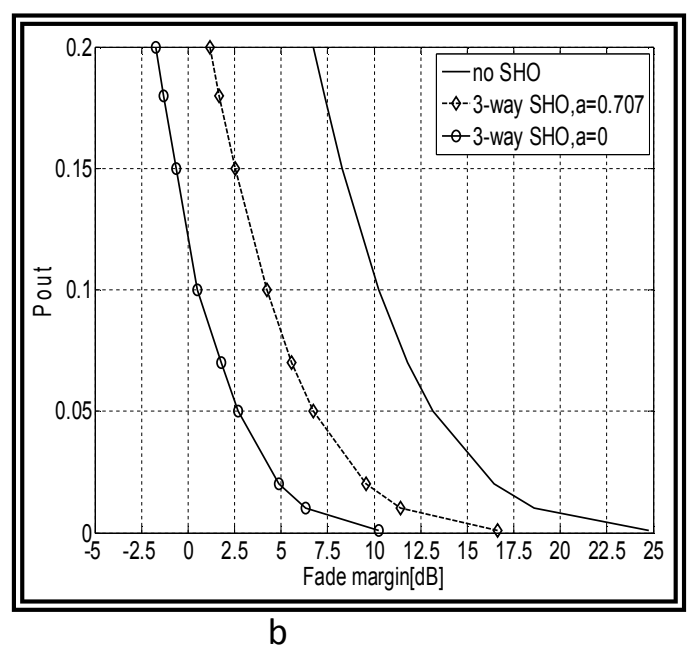

$\mathrm{b}$

Figure 5 Outage probabilities with fade margin when the MS is at the cell corner

Figure $(6 a, b)$ illustrates the required fade margin when the user moves away towards the cell corner, for no SHO and 2-way SHO when Pout $=0.1$. For $r / R=0.75$ with 2-way SHO the reduction in fade margin is about $3.2 \mathrm{~dB}$ and $1.3 \mathrm{~dB}$ for $\mathrm{a}^{2}=0$ and $\mathrm{a}^{2}=0.5$ respectively. Figure $6 \mathrm{~b}$ illustrates the required fade margin for 3-way $\mathrm{SHO}$. For $\mathrm{r} / \mathrm{R}=0.75$ the required fade margin without SHO is $5.2 \mathrm{~dB}$, while for 3 -way SHO is $0.27 \mathrm{~dB}$ and $1.3 \mathrm{~dB}$ for $\mathrm{a}^{2}=0$ and $\mathrm{a}^{2}=0.5$ respectively.

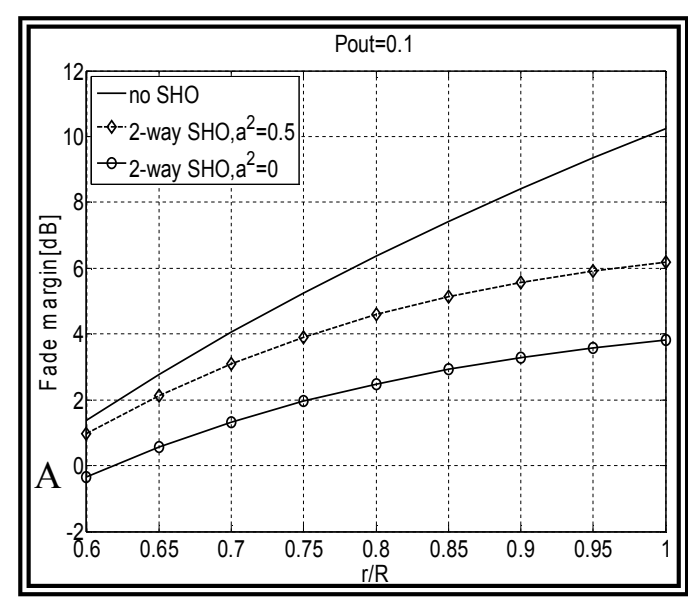

a

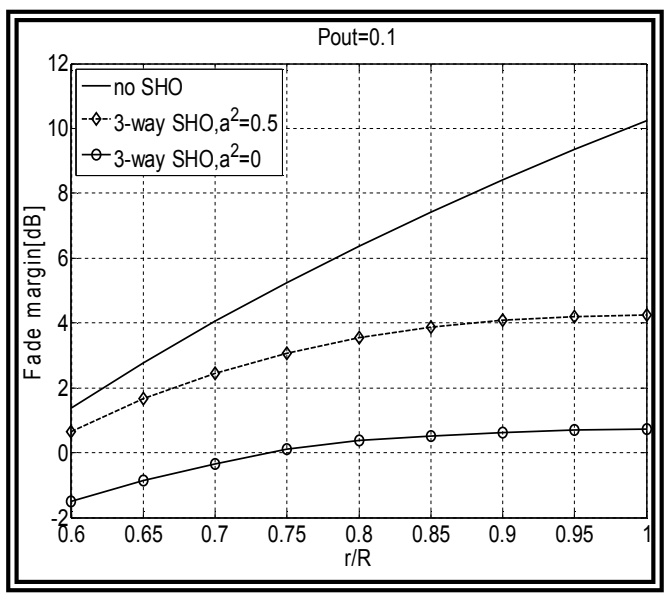

$\mathrm{b}$

Figure 6 Fade margin required when outage probability $=0.1$

Figure (7) illustrates the micro diversity gain when the user moves away towards the cell corner for 2-way SHO and 3-way SHO. for the MS is at the cell corner, with 2-way SHO the required $\mathrm{E}_{\mathrm{b}} / \mathrm{I}_{\mathrm{o}}$ is $3 \mathrm{~dB}$ lower compared without $\mathrm{SHO}$, while with 3-way $\mathrm{SHO}$ the required $\mathrm{E}_{\mathrm{b}} / \mathrm{I}_{\mathrm{o}}$ is $3.2 \mathrm{~dB}$ lower compared without $\mathrm{SHO}$ in order to obtain the same quality of service( $\mathrm{QoS})$ as shown in figure 8. The 3-way SHO has a slight improvement $(0.2 \mathrm{~dB})$ in comparison w Figure 8 illustrates the BER versus average SNR for no SHO, 2-way SHO, and 3-way SHO. From [13] the required BER for voice service is $10^{-3}$, to obtain this value, the required 
SNR without SHO is $24 \mathrm{~dB}$, while with 2-way SHO is $12.1 \mathrm{~dB}$, and with 3-way SHO is $7 \mathrm{~dB}$. It can be concluded that SHO decreases the average SNR by approximately $50 \%$ and $70 \%$ for 2-way SHO and 3-way SHO respectively when BER $=10^{-3}$. For data service the required BER is $10^{-4}$, for no SHO the required SNR is $33 \mathrm{~dB}$, for 2-way SHO is $17 \mathrm{~dB}$, and for 3-way SHO is $10 \mathrm{~dB}$. ith 2-way SHO as shown in the figure.

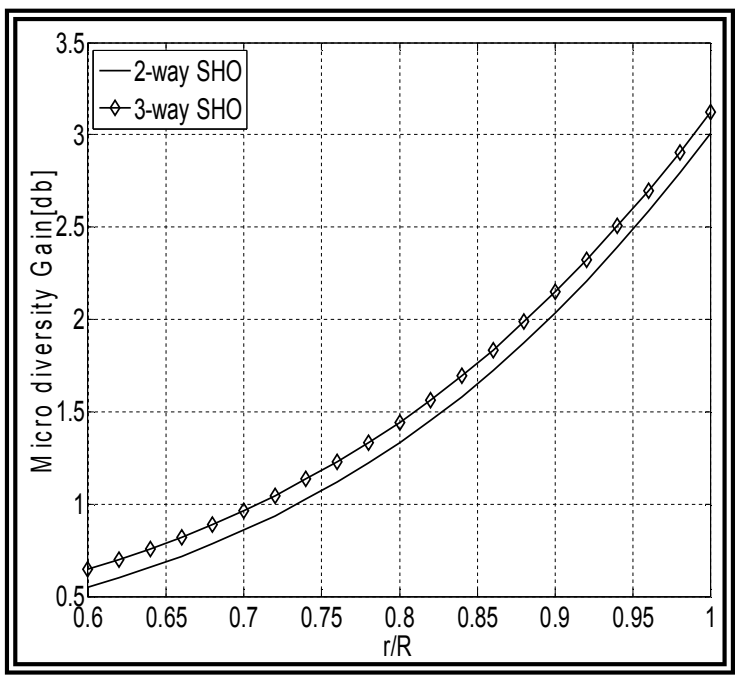

Figure (7) Micro diversity gain versus normalized distance

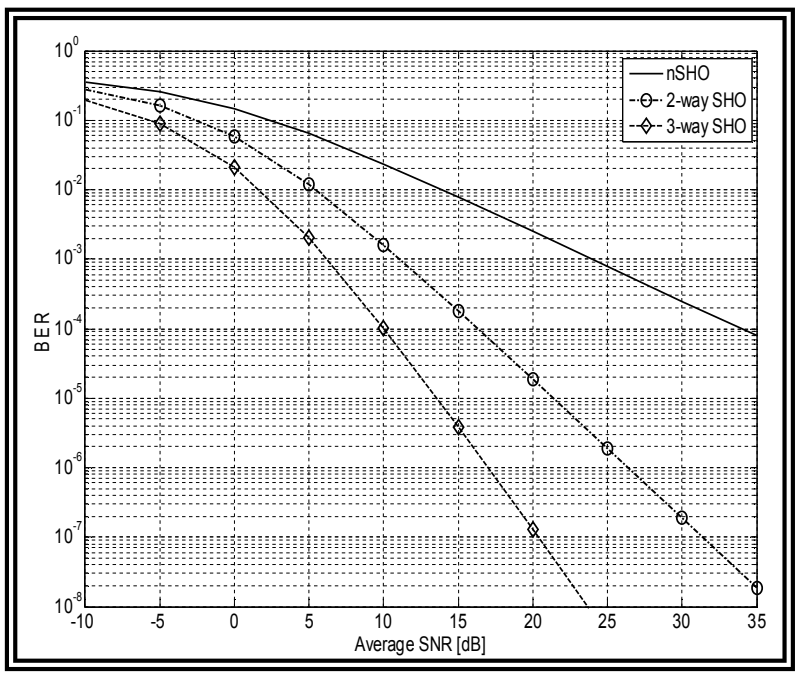

Figure 8 BER versus average SNR

Figure 9a illustrates the effect of path difference between two BSs on the average SNR. For a fixed BER, (e.g. $10^{-3}$ ), increasing the path difference to $3 \mathrm{~dB}$ will increase the average $S N R$ to $1.5 \mathrm{~dB}$ compared with the case path difference $=0 \mathrm{~dB}$, in order to obtain the same BER. Figure $9 \mathrm{~b}$ illustrates the effect of path difference between three BSs on the average SNR.
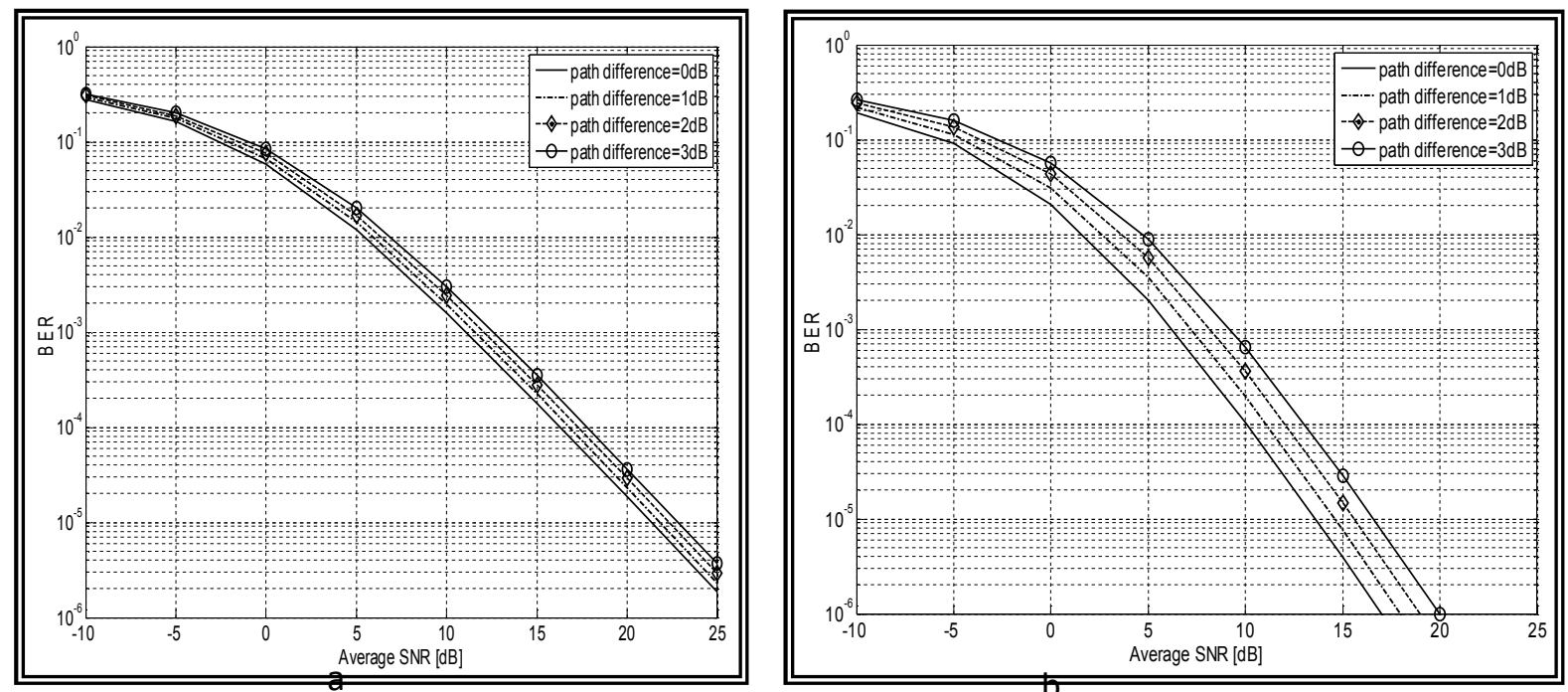

Figure 9 BER versus average SNR 


\section{Conclusions :}

The following conclusions can be drawn:

- For perfect power control $(\mathrm{PCE}=0 \mathrm{~dB})$ the $\mathrm{MS}$ needed less power comparing with imperfect power control in order to obtain same bit rate.

- WCDMA systems are interference limited, increasing PCE will increase the power consumption by MS which leads to increase the interference and decrease capacity (number of users per cells).

- For 3-way SHO the MS consumes more power (about 23\% to 47\%) compared to 2-way $\mathrm{SHO}$, while the number of users per cell for 3-way $\mathrm{SHO}$ is larger than (about $1 \%$ to $5.4 \%$ ) compared to 2-way SHO. So, 2-way $\mathrm{SHO}$ is recommended than 3-way SHO avoiding the complexity in implementation of 3-way soft handover.

- The fade margin decreased from $0.4 \mathrm{~dB}$ to $9.5 \mathrm{~dB}$, when $\mathrm{SHO}$ is implemented which leads to increasing coverage area of the cell.

- During SHO the MS needs a lower S/N to obtain the specific BER. For voice service, 2-way SHO decreases the S/N by $11.9 \mathrm{~dB}$ and 3 -way SHO by $17 \mathrm{~dB}$ while, for data service 2-way SHO decreases the S/N by $14 \mathrm{~dB}$ and 3-way SHO by $23 \mathrm{~dB}$.

\section{REFERENCES:}

[1]. N. Sori "Handoff Initiation and Performance Analysis in CDMA Cellular Systems", MSc. Thesis, Addis Ababa University, Feb. 2007.

[2]. I. A. Tomic, and M. L. Dukic, "Soft Handover and Downlink Capacity in UMTS Network", IEEE, 2004.

[3]. A. A. Al-Douri, " Soft Handover in WCDMA System", MSc. Thesis, University of Mosul, 2009.

[4]. S. Tamilselvan, N. T. Naidu and K. Manivannan, "Optimization of SHO Margin in WCDMA Network", IEEE Transaction on Vehicular Communications, 2008.

[5]. R. A.Cheema, and M. J. Irshad "Issues and Optimization of UMTS Handover", MSc. Thesis, Blekinge Institute of Technology, 2008.

[6]. R. Patachainanda, and K. Sandrasegrana, "System-Level Modeling and Simulation of Uplink WCDMA", IEEE Fifth International Conference on Information Technology, PP 1071-1076, 2008.

[7]. Y. Chen, "Soft Handover Issues in Radio Resource Management for 3G WCDMA Network", Doctoral Thesis, Queen Mary University of London, September 2003.

[8]. N. P. Singh, and B. Singh, "Effect of Soft Handover Margin under various Radio Propagation Parameters in CDMA Cellular Networks", IEEE Transaction on Vehicular Communications, PP 1-4, 2007.

[9]. A. J. Viterbi, " CDMA: Principles of Spread Spectrum Communication", AddisonWesley, 1995. 
[10]. T. S. Rappaport, "Wireless Communication Principles \& Practice", Prentice Hall PTR, Second Edition 2002.

[11]. H. Holma and A. Toskala "WCDMA for UMTS: Radio Access for Third Generation Mobile communications", John Wiley \& Sons, Ltd, Fourth Edition, 2007.

[12]. S.H.Y.Al-Bajary, "Performance Evaluation of Soft Handoff in WCDMA System" , MSc. Thesis, University of Mosul, 2010.

[13]. J. F. Monserrat, D. G. Barquero, D. Calabuig, L. Rubio, and N. Cardona "Evaluation of Soft Handover Micro Diversity Gain on the UMTS System Capacity and QoS", IEEE International Symposium on Personal, Indoor and Mobile Radio Communications, 2006.

[14]. D. Wong, and T.J.Lim, "Soft Handoffs in CDMA Mobile Systems", IEEE Personal Communications, pp. 6-16, 1997.

[15]. M. C. Fernandez, and H. M. AL-Housami, "Calculation of Soft Handoff Gain for UMTS",3G Mobile Communication Technologies IEE, PP 42-46, 2002.

[16]. J. E. R. Lima, "Comparative Study of Fade Margins with Hard and Soft Handoff in CDMA based Microcellular Systems", IEEE Transaction on Vehicular Communications, 2009.

[17]. Z. Mobini, MA. Mohammadi, H. A. Rohi, and M. Ardebilipour "Power Controlled Uplink Performance Analysis under Soft Handover in DS-CDMA Systems along Convontional Coding Scheme", IEEE International Colloquium on Communication, PP 518-522, 2008.

[18]. K. Heck, D. Steahle, and K. Leibnitz, "Diversity Effect on the Soft Handover Gain in UMTS Networks", IEEE Transaction on Vehicular Communications, PP 1269-1273, 2002. 